\title{
Changes in intestinal permeability and epithelial differentiation during inflammation in the rat
}

\author{
J K RAMAGE, R H HUNT, AND MARY H PERDUE \\ From the Intestinal Disease Research Unit, Departments of Pathology and Medicine, McMaster University, \\ Hamilton, Ontario, Canada
}

SUMmaRY We examined changes in gut permeability in a controlled model of inflammation produced in rats after infection with the nematode parasite, Nippostrongylus brasiliensis. The probe, ${ }^{51} \mathrm{Cr}$-EDTA, was injected into ligated loops of jejunum in vivo and recovery of radioactivity was measured in urine, kidney, and intact loop at five hours. Urinary recovery was significantly increased during the early (day 7) and acute (day 10) stages of the infection compared with values in control rats but subsequently returned to normal. Urinary clearance of the probe after iv injection was unaltered during infection. Villus atrophy occurred only at the acute stage, whereas crypt hyperplasia was evident at both the early and acute stages. The terminal ileum appeared normal and showed normal permeability when compared with controls. We conclude that permeability changes are local to the site of inflammation, are reversible after healing and may be related to an increase in the proportion of relatively undifferentiated epithelium.

\begin{abstract}
${ }^{51} \mathrm{Cr}$-EDTA (chromium-labelled ethylenediaminetetraacetate) has been introduced as an inert probe for assessing permeability of the intestine in patients with inflammatory bowel disease, coeliac disease and acute gastroenteritis. ${ }^{1-4}$ The probe is usually given orally and urinary excretion measured, hence little is known about the site of increased permeability in these inflammatory conditions. In addition, little is known about the temporal relationship of increased epithelial permeability to the acute and healing phases of the inflammation. If the urinary excretion does indicate relapses and remissions, the urinary recovery of the probe may become a useful way of monitoring the course of inflammatory diseases.

Nippostrongylus brasiliensis is an intestinal parasite of the rat which causes gross inflammation of the jejunum between seven and 14 days after subcutaneous inoculation of larvae. Villus atrophy, crypt hyperplasia and mucosal inflammatory infiltrates are the main features of the intestinal lesion which starts to heal after worm expulsion at approximately 14 days. ${ }^{5}$ In preliminary experiments administering ${ }^{51} \mathrm{Cr}$ EDTA by gavage to rats we showed that urinary

Address for correspondence: Dr Mary Perdue. Intestinal Disease Research Unit, 3N5. Health Sciences Centre, McMaster University, 12(k) Main Street West, Hamilton, Ontario, Canada. I.8N 37.5

Received for publication 17 June 1987.
\end{abstract}

recovery was increased at day 7 and 10 after infection compared with controls. In the current studies we used a ligated loop technique to more accurately measure permeability changes. We assessed mucosal histology and enzyme activities during the course of the infection in order to relate permeability changes to alterations in the epithelium.

\section{Methods}

ANIMALS

Male Sprague-Dawley rats (Charles River Breeding Laboratories, Wilmington, MA) weighing $275-325 \mathrm{~g}$ were infected by subcutaneous injection of 3000 larvae of Nippostrongylus brasiliensis in $0.5 \mathrm{ml}$ phosphate-buffered saline. ${ }^{5}$ Rats were housed with a constant temperature and lighting cycle. Animals were studied at day 7 (early), 10 (acute), and 21 (recovery) of the infection. Controls consisted of non-infected litter mates.

\section{HISTOLOGY}

Animals were killed by cervical dislocation and the gut rapidly removed. Two one centimetre segments of gut $18 \mathrm{~cm}$ distal to the ligament of Treitz were opened longitudinally, placed flat on a piece of card which was then placed in fixative. Formalin was used 
to fix for routine staining with haematoxylin and eosin and Carnoy's fixative for toluidine blue staining (for identification of intestinal mucosal mast cells). ${ }^{6}$

BIOCHEMICAL, DETERMINATIONS

The segments of gut for enzyme and histamine assays were obtained from the region between 20 and $30 \mathrm{~cm}$ distal to the ligament of Treitz. These were divided into $3 \mathrm{~cm}$ segments for thymidine kinase assay and $7 \mathrm{~cm}$ segments for other assays. Both segments were flushed with saline, opened longitudinally, blotted and weighed and then the mucosa was scraped for the underlying muscle between two glass slides. The scrapings were weighed, homogenised in buffer (sucrose buffer for thymidine kinase assays and EDTA for other assays) and the samples were frozen in acetone and dry ice and stored at $-70^{\circ} \mathrm{C}$. Samples for histamine assay were homogenised, boiled for 10 minutes, centrifuged and the supernatant frozen and stored in the same way. The samples were analysed for histamine, protein, sucrase, maltase, alkaline phosphatase and thymidine kinase as described. ${ }^{*}$

PERMEABILITY

Animals were anaesthetised with ip pentobarbitone. A tracheostomy was carried out and a polyethylene cannula (PE50) inserted into the right jugular vein under direct vision. A continuous infusion of pentobarbitone and saline $(2.4 \mathrm{ml} / \mathrm{h})$ was delivered through this cannula to maintain anaesthetic state and good hydration. Throughout the experiment the temperature of the animals was maintained between $37^{\circ}$ and $40^{\circ} \mathrm{C}$ by a heating pad which was thermostatically controlled by link to a rectal thermometer. A laparotomy was carried out and a purse string suture inserted in the fundus of the bladder through which a shouldered silastic cannula was inserted. A further stay suture was tied around the cannula distal to the shoulder avoiding the ureters. A syringe was attached to the other end of the cannula and urine was collected during the five hour experiment. The urethra was isolated and ligated to prevent any loss of urine.

A $15 \mathrm{~cm}$ loop of jejunum starting $3 \mathrm{~cm}$ distal to the ligament of Treitz was carefully isolated and ligated proximally without interfering with the blood supply. At the distal end the same procedure was followed except that before the ligature was tightened a shouldered silastic cannula was inserted which was held in place with two fine sutures. (Preliminary studies using injection into the loop with a fine needle showed significant leakage from the needle puncture site.) The probe, $1.8 \times 10^{5} \mathrm{~Bq}(5 \mu \mathrm{Ci})$ of ${ }^{51} \mathrm{Cr}$-EDTA $(0.1 \mathrm{M})$ made up to $1.6 \mathrm{ml}$ in $5 \%$ dextrose $(\mathrm{pH} 5$, osmolality 330, prepared by Hamilton and District Radiopharmacy, McMaster University, Hamilton,
Canada), was gradually injected into the loop and left in place for five hours. The abdomen was closed in two layers with silk. At five hours after injection of the probe, the urine was collected and the bladder washed out twice with $0.5 \mathrm{ml}$ of distilled water, the washings being added to the original urine. Samples were counted for five minutes in a well type gamma counter (1282 Compugamma, LKB WALLAC, Fisher Scientific, Toronto, Canada) and the counts compared with the counts of known volumes of previously prepared standards of the administered ${ }^{51} \mathrm{Cr}$-EDTA. The left kidney and the jejunal loop were also removed and counted. Similar experiments were done to study the permeability of the ileum in $15 \mathrm{~cm}$ loops ending $2 \mathrm{~cm}$ proximal to the ileocaecal valve. To assess renal function during intestinal inflammation $3 \cdot 7 \times 10^{3} \mathrm{~Bq}(0 \cdot 1 \mu \mathrm{Ci})$ of the probe was injected iv in control and day 10 infected animals and the urine similarly collected and counted.

\section{STATISTICS OF ANALYSIS}

All analyses were carried out by a one-way analysis of variance for overall effect between day of infection followed by unpaired $t$ test (using the pooled variance of all four groups) for individual differences from controls. Some of the enzyme data showed heterogeneous variance between days of infection and this data was log transformed and retested. These tests gave the same $p$ values as the data presented.

\section{Results}

HISTOLOGY

The mean villus height and crypt depth determined from sections of jejunum from control rats and from rats during various stages of inflammation are shown in Figure 1. The initial abnormality, crypt hyper-

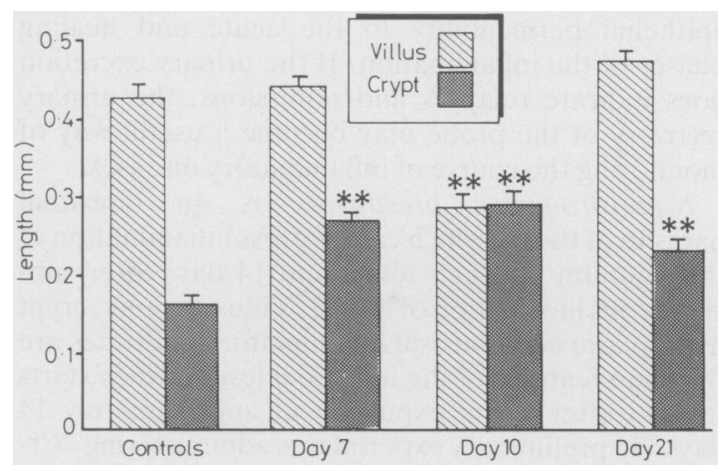

Fig. 1 Villus height and crypt depth in control and infected rats. Values represent mean $\pm S E M$. At least three welloriented villus/crypt units were measured in each section. ${ }^{* *}=p<0 \cdot 01$. Number of animals at each time was: controls, 8; day 7, 6; day 10,8; day 21,8 . 
Table 1 Villus/crypt ratios and mast cell numbers in sections from control and infected rats

\begin{tabular}{llcll}
\hline & Control & Day 7 & Day 10 & Day 21 \\
\hline $\mathrm{n}$ & 6 & 7 & 6 & 6 \\
Villus/crypt & $2 \cdot 79 \pm 0 \cdot 11$ & $1 \cdot 73 \pm 0 \cdot() 6$ & $1 \cdot 02 \pm 0 \cdot 08$ & $2 \cdot(05 \pm 0 \cdot 09$ \\
$\mathrm{p}$ & & $<0 \cdot 05$ & $<0 \cdot 01$ & $\mathrm{NS}$ \\
Mast cells & $3 \cdot 1 \pm 0 \cdot 2$ & 0 & $3 \cdot 0 \pm 1 \cdot 2$ & $>40$ \\
\hline
\end{tabular}

Values represent mean $\pm \mathrm{SEM} ; \mathrm{n}=$ number of sections evaluated; p, compared to control values; $N S=$ not significant. In each section measurements were made on at least three well oriented villus crypt units. Mast cells are expressed per villus crypt unit; at day 21 mast cells were too numerous to be counted accurately. Sections for mast cell counting were fixed in Carnoy's fixative and stained with toluidine blue.

Table 2 Enzyme activities and histamine levels in jejunal mucosal homogenates

\begin{tabular}{lcccc}
\hline & Control & Day7 & Day 10 & Day 21 \\
\hline n & 8 & 6 & 10 & 8 \\
Maltase & $189 \pm 31$ & $243 \pm 38$ & $159 \pm 25$ & $267 \pm 37$ \\
Sucrase & $47 \cdot 0 \pm 7 \cdot 5$ & $61 \cdot 9 \pm 9 \cdot 5$ & $32 \cdot 3 \pm 4 \cdot 1^{*}$ & $65 \cdot 1 \pm 7 \cdot 5$ \\
Alk phos & $0 \cdot 52 \pm 0 \cdot 10$ & $0 \cdot 29 \pm 0 \cdot 05$ & $0 \cdot 17 \pm 0 \cdot 02 \dagger$ & $0 \cdot 30 \pm 0 \cdot 04$ \\
Tkase & $5 \cdot 1 \pm 1 \cdot 5$ & $22 \cdot 8 \pm 6 \cdot 4^{*}$ & $21 \cdot 0 \pm 4 \cdot 4^{*}$ & $2 \cdot 6 \pm 0 \cdot 7$ \\
Histamine & $11 \cdot 8 \pm 3 \cdot 1$ & $2 \cdot 5 \pm 0 \cdot 5^{*}$ & $12 \cdot 8 \pm 1 \cdot 8$ & $211 \cdot 8 \pm 32 \cdot 8 \dagger$ \\
\hline
\end{tabular}

Values of sucrase, maltase, alkaline phosphatase (Alk phos) and thymidine kinase (T kase) are expressed as U/g protein; histamine is expressed as $\mu \mathrm{g} / \mathrm{g}$ protein. Means $\pm S E M$ are shown; $n=$ number of animals; ${ }^{*} p<0.05,+p<0.01$ compared with controls ( $p$ values for $t$ test given only if ANOVA $p<0 \cdot 05$ ).

plasia, was evident by day 7 and continued at day 10 and day 21. Villus height was normal at day 7 ; however, by day 10 villus atrophy was observed. Villus height had returned to normal at day 21. Similar changes were not observed in the terminal ileum of day 10 infected animals. Intestinal mucosal mast cell numbers and villus/crypt ratios are shown in Table 1 . The villus/crypt ratio was significantly reduced in sections from infected rats at 7 and 10 days but was not different from controls at day 21 . In control rats jejunal mucosal mast cell numbers were consistently low. At day 7 no stained mast cells were visualised; at day 10 mast cells were again apparent; at day 21 mast cell numbers were increased compared to controls and to the earlier stages of infection.

\section{BIOCHEMICAL DETERMINATIONS}

Mean activities of maltase, sucrase, alkaline phosphatase and thymidine kinase, and mean histamine levels in controls and the three stages of infection are shown in Table 2. The activities of the brush border enzymes, sucrase and alkaline phosphatase, were decreased only at day 10 . Thymidine kinase activity, an enzyme involved in DNA replication, was increased at both day 7 and day 10 but was normal at

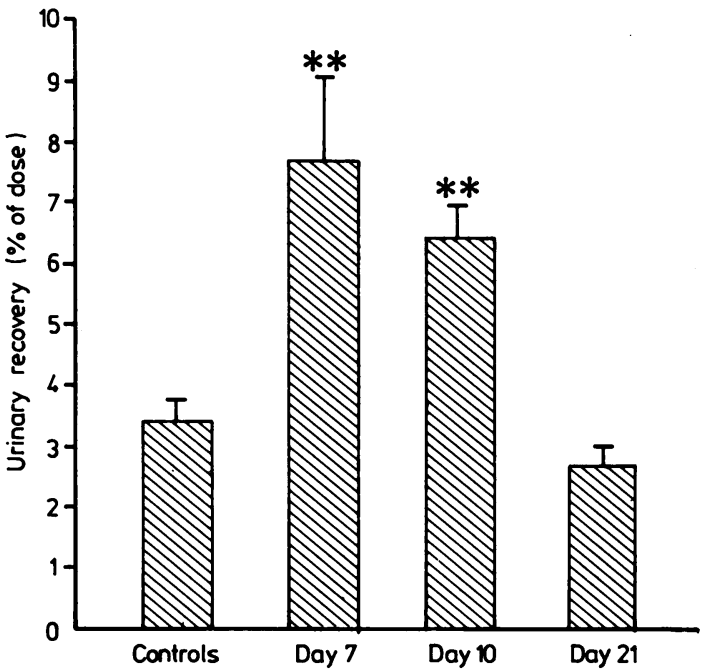

Fig. 2 Urinary recovery of ${ }^{51} \mathrm{Cr}-\mathrm{EDTA}$ after injection into jejunal loops in control and infected rats. Bars represent mean $\pm S E M, \%$ administered dose ${ }^{* *}=p<0.01$. Number of animals at each time was: controls, 8; day 7, 6; day 10, 8; day 21,8 .

day 21. Histamine concentrations were decreased at day 7, normal at day 10 and significantly increased at day 21 , a similar pattern to the mast cell counts and consistent with previous studies. ${ }^{9}$

\section{PERMEABILITY}

Urinary recovery of ${ }^{\text {si }} \mathrm{Cr}$-EDTA, expressed as a mean percentage of the original administered dose, after injection into jejunal loops is shown in Figure 2. Both day 7 and day 10 animals had significantly increased recovery compared with controls. Although an increased glomerular filtration rate is unlikely to be present in an intestinal parasitic infection we tested this possibility by iv injection of the probe in three control and three infected animals. Five hour urinary recovery in controls and day 10 rats was not significantly different $(83.61 \pm 2 \cdot 15 \quad v$ $84 \cdot 24 \pm 0 \cdot 88$, mean \pm SEM, \% of administered dose, respectively). Recovery of the probe from the left kidney and from the intact loop is shown in Table 3.

Table 3 Recovery of ${ }^{51} \mathrm{Cr}$-EDTA from kidney and intact jejunal loop

\begin{tabular}{lcclc}
\hline & Control & Day 7 & Day 10 & Day 21 \\
\hline n & 8 & 6 & 8 & 8 \\
Kidney & $0 \cdot 09 \pm 0 \cdot 03$ & $0 \cdot 18 \pm 0 \cdot 03^{*}$ & $0 \cdot 14 \pm 0 \cdot 05$ & $0 \cdot 08 \pm 0 \cdot 02$ \\
Jejunal loop & $68 \cdot 5 \pm 4 \cdot 1$ & $74 \cdot 0 \pm 3 \cdot 9$ & $73 \cdot 6 \pm 5 \cdot 6$ & $76 \cdot 3 \pm 2 \cdot 0$ \\
\hline
\end{tabular}

Values represent mean $\pm \mathrm{SEM}, \%$ administered dose $; n=$ number of animals; ${ }^{*} p<0 \cdot 05$. 
Table 4 Recoveryof of ${ }^{51} \mathrm{Cr}$-EDTA after injection into ileal loops

\begin{tabular}{lcc}
\hline & Control & Infected (day 10) \\
\hline $\mathrm{n}$ & 5 & 5 \\
Urine & $0 \cdot 81 \pm 0 \cdot 08$ & $0 \cdot 97 \pm 0 \cdot 18$ \\
Kidney & $0 \cdot 03 \pm 0 \cdot 01$ & $0 \cdot 04 \pm 0 \cdot 01$ \\
Gut & $79 \cdot 8 \pm 2 \cdot 32$ & $70 \cdot 18 \pm 4 \cdot 87$ \\
\hline
\end{tabular}

Values are expressed as mean $\pm \mathrm{SEM}, \%$ of administered dose. No significant differences were seen between the groups.

Recovery from the kidney followed the same pattern as the urine; recovery from the intestinal loop did not change significantly during the course of the infection. Table 4 shows urinary recovery after injection into ileal loops in control and day 10 infected animals. Recovery of the probe from the ileum was unaltered by the more proximal inflammation.

\section{Discussion}

We have shown a significant increase in urinary recovery of ${ }^{51} \mathrm{Cr}$-EDTA from the jejunum during the early and acute stages of the inflammation caused by Nippostrongylus brasiliensis. This increase did not occur in the terminal ileum, a site distal to the inflammation. Our finding that urinary recovery of ${ }^{51} \mathrm{Cr}$-EDTA after iv administration was unaltered by intestinal inflammation indicates that the changes we observed were because of an increase in epithelial permeability rather than changes in renal function. By day 21, after worm expulsion, the permeability defect had returned to normal. Increased gut permeability appeared to be associated with changes in the crypt rather than with villus atrophy: at day 7 we found permeability changes with no alteration in the villus height. Crypt hyperplasia was evident at this early stage, along with a rise in the thymidine kinase activity, indicating that increased crypt cell proliferation was an early effect of the infection and subsequent inflammation. Increased permeability at this stage suggests that a less differentiated epithelium may be more permeable than a normal epithelium to the probe. Permeability to another inert probe, lactulose, has been reported to correlate closely with crypt hyperplasia in chronic diarrhea. ${ }^{10}$ Decreased 'tightness' (based on strand number) of the tight junctions in the crypt compared with the villi has been described. " Alternatively, increased sloughing at the villus tips due to faster cell turnover or altered pinocytosis in undifferentiated crypt like enterocytes may explain our findings. Although crypt length was still increased at day 21 when urinary recovery of the probe had returned to normal, villus/crypt ratios and enzyme activities, including thymidine kinase activity, had returned to control values. This suggests that increased crypt length alone is not an accurate indication of undifferentiated epithelium. Others have also reported this finding. ${ }^{12} 13$

Increased permeability might be considered to be directly related to the action of the worms themselves on the epithelium. From our experiments we cannot exclude this possibility. Previous experiments in T cell/mast cell deficient rats showed that although Nippostrongylus brasiliensis worms invaded the gut, morphological abnormalities were absent. ${ }^{14}$ In a separate study, macromolecular permeability changes disappeared with cortisone treatment even though the worms persisted in the jejunum. ${ }^{6}$ In addition, intestinal permeability changes have been described in other inflammatory conditions not produced by infection. ${ }^{315}$ It is thus likely that the increased permeability during inflammation in our model is related to some aspect of changes in the epithelium caused by the cell mediated immune reaction to the parasite.

Numbers of stained mast cells and histamine concentrations in the lamina propria were decreased at day 7 suggesting that mast cells may have degranulated in response to the parasite. It is possible that mast cell mediators affect enterocyte proliferation. Mast cells are only one component of the inflammatory response, however, and other immune cells and mediators may be involved. At day 21 the numbers of mast cells in the lamina propria were greatly increased. This phenomenon appears to be associated with the ability of the 'immune' rat to expel worms rapidly upon secondary challenge. ${ }^{16} \mathrm{At}$ day 21 , however, gut permeability was normal. Therefore, permeability changes cannot be related to the actual numbers of mast cells present. At this stage the worms have been expelled and mast cells would not be expected to degranulate unless re-exposure to worms or worm antigen occurred.

Information from studies examining changes in the inflamed mucosa associated with changes in permeability to ${ }^{51} \mathrm{Cr}$-EDTA in animal models is important for understanding the changes documented in clinical studies. Extensive clinical data have already been collected using this probe. The possibility that increased permeability occurs across a relatively undifferentiated epithelium may eventually increase our understanding of mechanisms in certain inflammatory diseases. Extrapolation of our findings to the transport of larger antigenic molecules is difficult, but in other experimental situations increased permeability to macromolecules follows that of smaller molecules. ${ }^{17}$ Indeed, our preliminary observations have shown that uptake of a 'bystander' 
protein molecule, ovalbumin, closely parallels the increased uptake of ${ }^{51} \mathrm{Cr}$-EDTA into the circulation. Further studies are warranted to define more accurately which aspects of epithelial damage are related to changes in permeability.

The authors would like to thank Ms Nancy Dyck and Mr Stephen Masson for excellent technical assistance and Dr J Bienenstock, Drs R T Jenkins and R Riddell for their advice. Preparation of ${ }^{51} \mathrm{Cr}$-EDTA and information from the staff of the Hamilton and District Radiopharmacy is gratefully acknowledged. This work was supported by grants from the Medical Research Council of Canada and the Canadian Foundation for Ileitis and Colitis.

This work has been presented in part at the meeting of the British Society of Gastroenterology, September 1986 and published in abstract form (Gut 1986; 27: A1261).

\section{References}

1 Bjarnason I, O’Morain C, Levi AJ, Peters TJ. Absorption of ${ }^{51}-\mathrm{Cr}$ labelled ethylenediamine-tetraacetate in inflammatory bowel disease. Gastroenterology 1983; 85: 318-22.

2 Jenkins RT, Goodacre RL, Reilly RM, Hunt RH, Bienenstock J. Intestinal permeability in Crohn's disease, ulcerative colitis and gluten-sensitive enteropathy. Gastroenterology 1985; 88: 1433.

3 Bjarnason I, Marsh MN, Price A, Levi AJ, Peters TJ. Intestinal permeability in patients with coeliac disease and dermatitis herpetiformis. Gut 1985; 26: 1214-9.

4 Forget P, Sodoyez-Goffaux F, Zapitelli A. Permeability of the small intestine to ${ }^{51} \mathrm{Cr}$-EDTA in children with acute gastroenteritis or eczema. J Pediatr Gastroenterol Nutr 1985; 4: 393-6.

5 Ogilvie BM, Jones VE. Nippostrongylus brasiliensis: a review of immunity and the host/parasite relationship in the rat. Exp Parasitol 1971; 29: 138-77.
6 Murray M, Jarrett WFH, Jennings FW. Mast cells and macromolecular leak in intestinal immunological reactions: the influence of sex of rats infected with Nippostrongylus brasiliensis. Immunology 1971; 21: 17-31.

7 Kelly MH, Butler DG, Hamilton JR. Transmissible gastroenteritis in piglets: A model of infantile diarrhea. J Pediatr 1972; 80: 925-31.

8 Perdue MH, Chung M, Gall DG. The effect of intestinal anaphylaxis on gut function in the rat. Gastroenterology 1984; 86: 391-7.

9 Befus AD, Johnson N, Bienenstock J. Nippostrongylus brasiliensis: mast cells and histamine levels in tissues of infected and normal rats. Exp Parasitol 1979; 48: 1-8.

10 Ford RPK, Menzies S, Phillips AD, Walker-Smith JA, Turner MW. Intestinal sugar permeability: relationship to diarrhoeal disease and small bowel morphology. J Pediatr Gastroenterol Nutr 1985; 4: 568-74.

11 Marcial MA, Carlson SL, Madara JL. Partitioning of paracellular conductance along the ileal crypt-villus axis: a hypothesis based on structural analysis with detailed consideration of tight junction-function relationships. J Membrane Biol 1984; 80: 59-70.

12 Symons LEA. Kinetics of the epithelial cells, and morphology of villi and crypts in the jejunum of rats infected by the nematode Nippostrongylus brasiliensis. Gastroenterology 1965; 49: 158-68.

13 Smith MW. Expression of digestive and absorptive function in differentiating enterocytes. Ann Rev Physiol 1985; 47: 247-60).

14 Bjarnason I, Smethurst P, Levi AJ, Peters TJ. Intestinal permeability to ${ }^{51} \mathrm{Cr}$-EDTA in rats with experimentally induced enteropathy. Gut 1985; 26: 579-85.

15 Ferguson A, Jarrett EEE. Hypersensitivity reactions in the small intestine. I. Thymus dependency of experimental 'partial villous atrophy'. Gut 1975; 16: 114-7.

16 Lee TDG, Swieter M, Befus AD. Mast cell responses to helminth infection. Parasitol Today 1986; 2: 186-91.

17 Ecknauer R, Buck B, Breitig D. An experimental model for measuring intestinal permeability. Digestion 1983; 26: $24-32$. 